University of San Diego

Digital USD

Spring 5-22-2021

\title{
Bridging the Gap: Utilization of Telehealth for Heart Failure Patients to Reduce Hospital Readmissions: Best Practice and Recommendations
}

Shalaine Corbilla

University of San Diego, scorbilla@sandiego.edu

Follow this and additional works at: https://digital.sandiego.edu/dnp

Part of the Nursing Commons

\section{Digital USD Citation}

Corbilla, Shalaine, "Bridging the Gap: Utilization of Telehealth for Heart Failure Patients to Reduce Hospital Readmissions: Best Practice and Recommendations" (2021). Doctor of Nursing Practice Final Manuscripts. 155.

https://digital.sandiego.edu/dnp/155

This Doctor of Nursing Practice Final Manuscript is brought to you for free and open access by the Theses and Dissertations at Digital USD. It has been accepted for inclusion in Doctor of Nursing Practice Final Manuscripts by an authorized administrator of Digital USD. For more information, please contact digital@sandiego.edu. 
Bridging the Gap: Utilization of Telehealth for Heart Failure Patients to Reduce Hospital Readmissions: Best Practice and Recommendations

UNIVERSITY OF SAN DIEGO

Hahn School of Nursing and Health Science

Beyster Institute of Nursing

\section{DOCTOR OF NURSING PRACTICE PORTFOLIO}

by

Shalaine Brooke Corbilla

A portfolio presented to the

FACULTY OF THE HAHN SCHOOL OF NURSING AND HEALTH SCIENCE UNIVERSITY OF SAN DIEGO

In partial fulfillment of the

requirements for the degree

DOCTOR OF NURSING PRACTICE

May 2021 


\begin{abstract}
Background: Heart failure is a leading cause of morbidity and mortality in the United States and accounts for a vast number of 30-day hospital readmissions. Within the first 30 days of discharge, patients and caregivers often misinterpret discharge instructions or lack access to providers. During this period, some modifiable symptoms become too difficult to tolerate and patients return to the emergency department for assessment and ultimately readmission for high-risk complications. The Sars-CoV-2 pandemic has added additional barriers for patients to receive care after a hospital discharge.

Purpose: To identify current best practices used for outpatient follow up with this patient population, develop a standardized intervention project for an advanced practice registered nurse (APRN) to use with heart failure patients, and make recommendations on how to implement the process within their practice.
\end{abstract}

Evidenced-based Intervention: A three-phase APRN intervention: (a) chart review of hospital course, disposition, and assessment of telemedicine follow up barriers, (b) a telehealth assessment within 1 week of discharge using a heart failure outpatient clinic telephonic consulting questionnaire to assess for progress or deterioration, and (c) a final 4-week assessment using the heart failure questionnaire to review progress and assess readiness for cardiac rehab enrollment.

Implications for Practice: Optimizing modifiable symptoms is paramount to successfully transitioning home from the hospital. Utilizing best practice will optimize modifiable symptoms for a successful transition home from the hospital by increasing access to care, exposure to frequent education and awareness to disease thus moving towards the goal of reducing hospital readmissions. 
Keywords: heart failure, transitions of care, telemedicine, advanced practice registered nurse, pandemic 
Bridging the Gap: Utilization of Telehealth for Heart Failure Patients to Reduce Hospital Readmissions: Best Practice and Recommendations

\section{Description of the Clinical Problem}

Heart failure is a leading cause of morbidity and mortality in the United States, affecting an estimated 5.7 million Americans (K. K. Lee et al., 2020). It is a brittle condition that requires close monitoring and frequent office visits. The unprecedented Sars-CoV-2 pandemic has increased barriers for heart failure patients to receive timely care after a hospital discharge. Social distancing requirements have led offices to function with minimal staffing, transitioning all applicable appointments to telemedicine. For some heart failure patients, a month follow up via telemedicine may be too late to assess deterioration. Patients and their caregivers now need more access to providers soon after a hospital discharge to discuss modifiable symptoms or complications such as medication adherence, activity levels, weight trends, and dietary compliance. Utilization of telemedicine and providing an appointment within seven days of discharge allow providers to mitigate these complications thus decreasing 30-day hospitalizations.

\section{Evidence for the Clinical Problem}

Since 2012, with the initiation of the Hospital Readmissions Reduction Program of the Patient Protection and Affordable Care Act, hospitals are faced with financial penalties for unacceptably high 30-day readmission rates (J. Lee et al., 2019). Heart failure is one of the six conditions or procedures specific 30-day risk-standardized unplanned readmission measures within the Medicare Hospital Readmissions Reduction Program thus making improving outcomes and decreasing hospitalizations a nationally recognized priority (Centers for Medicare \& Medicaid Services, 2020). The American 
Heart Association (2018) notes multiple, typical breakdowns leading to hospital readmissions. Factors for readmission include patient assessment breakdowns, family caregiver education breakdowns, handoff communication breakdowns, and breakdowns following discharge. In addition to usual barriers, the novel Sars-CoV-2 pandemic has made in person follow up access an additional obstacle to care. Despite continued efforts by care providers, readmission rates remain high.

\section{Purpose}

The aim of this evidenced based practice (EBP) project is to identify methods currently used to facilitate patient follow up after a hospital discharge prior to and during a pandemic and select one of the evidence-based methods to implement an advanced practice registered nurse (APRN) transitions-of-care access and month-long follow-up telehealth access to patients recently discharged from the hospital. Utilizing the services of a nurse practitioner student, patients would enroll in a discharge follow-up program that would increase their access to a provider. The project plan would be proposed to heart failure teams and cardiologists at a later date for potential use with their patient populations. No actual patient data were collected for this phase of the project. Data will be collected and analyzed to assess whether having a standardized transitions-of-care program would reduce 30-day hospital readmissions.

\section{EBP Model and Framework}

This project employed the five steps of EBP (Straus et al., 2019). The project began by asking the clinical question: What does it take to reduce heart failure 30-day hospital readmissions? Following the steps of the framework involved acquiring best practices, establishing an evidenced based intervention, and formulating a project 
process. As a nationally recognized priority, determining a need for improvement was already established. Acquiring evidence in support of an intervention determining what percentage of patients had access to care and, of those patients, how many were readmitted to the hospital. Important inquiries to support the intervention included what was already used by the current population, establish resources within the population, and the clinics that could accommodate the transition for telemedicine quickly. Outcomes and findings will be presented to hospitals and outpatient providers to improve their clinical plan of care and broaden access for their patients.

\section{Evidenced Based Intervention Identification}

A review of literature was completed using the following search engines: Cochrane, PubMed, and CINAHL. Keywords utilized with each search engine were readmission, heart failure, transitions of care, pandemic, cost, advanced practice registered nurse, and telemedicine. This search yielded over 5,500 articles from the past 5 years in peer-reviewed publications. Articles were ranked using the Johns Hopkins EBP model (Dang \& Kearholt, 2017). Current practices identified for post hospital discharge follow up were usual practice: in person visits, outcomes that were not reviewed for this project; virtual visits; structured telephone support; and transitional care clinics. Ten articles were chosen after extensive review of the article search article.

In response to the pandemic and new limitations for follow up, Wosik et al. (2021), conducted a retrospective study reviewing encounters from March 2020 and June 2020 and compared 30-day outcomes from the same period in 2019. In-person visits were 33,097 in $2019 ; 2,045$ patients, or $6.2 \%$ of these patients experienced emergency department (ED) visit or were hospitalized within 30-dayof their primary discharge. For 
the same time in 2020, overall encounters were 22,156, including 4,384 telehealth visits. The number of ED visits or hospitalizations within 30 days were 946 (5.4\%) and 172 (3.9\%) respectively. These authors reported a $33.1 \%$ decrease in the number of outpatient visits conducted during the first 15 weeks of the pandemic and a higher percentage (53\% versus $35 \%$ in 2019) of cancelled visits. Lastly younger, healthier patients utilized telehealth (Wosik et al., 2021). Results of their study suggested that patients who utilized telehealth were less likely to have an emergency visit or hospitalization when compared to those who had in-person visits. Although this study did not include visits for posthospital encounters, it was useful for this DNP project to demonstrate decreased incidence of ED visits or hospitalization when utilizing telemedicine.

The Sars-CoV-2 pandemic posed numerous barriers for heart failure patients. Tersalvi et al. (2020) reviewed telemedicine strategies and provided an overview of its application during a pandemic. These authors suggested that the benefit of virtual visits included the ability to provide access to patients who would otherwise have difficulty making appointments, titrate medications, and optimize stable patients but were inadequate in assessing volume status or issues that would otherwise be noted with an inperson exam. Under different circumstances, telemedicine had its limitations. One significant barrier was patients who did not have access to a telephone, the internet, or hardware services. Other limitations include old age, poor hearing, cognitive dysfunction, and language barriers. For patients with significant limitations and technological access, telephone support was suggested as the most readily applicable contact method and could be performed by trained personnel. Tersalvi and colleagues concluded that a costefficient, user-friendly, and person-centered system might not show improved outcomes, 
but it was not inferior to traditional ways, thus allowing a safe alternative to the status quo.

The objective of the study by Blum et al. (2020) was to assess the costeffectiveness of three types of post-discharge heart failure transitional care services with standard of care. These methods included a disease management clinic, a nurse home visit, and nurse case management compared to standard care. Blum and colleagues concluded that all three alternative methods were effective in reducing costs, all-cause mortality, and rehospitalizations, but increased overall lifetime health care costs secondary to increased life expectancy.

In response, The Heart Failure Readmission Intervention by Variable Early Follow-up (THRIVE) study demonstrated that early follow-up with structured telephone support could increase 7-day follow up and reduce in-person visits with comparable 30day clinical outcomes (K. K. Lee et al., 2020). A randomized trial assigned patients to an initial, structured telephone visit with a nurse or pharmacist or an initial in-person clinic appointment. A total of 2,091 patients were randomly assigned into a study arm. Their primary study outcome was reduced hospital readmissions with a result of $10.6 \%$ readmission rate for initial physician clinic follow up and $8.6 \%$ for nonphysician telephone-guided follow up (K. K. Lee et al., 2020).

Feltner et al. (2014) performed a systematic review to assess the efficacy, comparative effectiveness, and harms of transitional care interventions to reduce readmission and mortality rates for adults hospitalized with heart failure. Two reviewers examined 47 trials and noted that high intensity home-visiting programs reduced allcause readmission rates at 30 days and both home-visiting programs and 
multidisciplinary heart failure clinic interventions reduced all-cause readmissions over 3 to 6 months (Feltner et al., 2014). For specific heart failure-related readmissions, home visiting programs, structured telephone support, and multidisciplinary clinic interventions were moderate- to high intervention resources that reduced heart failure-specific readmissions.

Negarandeh et al. (2019) evaluated self-care behaviors and readmission in heart failure patients after discharge using telephone monitoring and usual patient care arms. A short 8 -week study evaluated 80 patients with a primary end point to assess heart failure self-care and related readmission rates. The European Heart Failure Self-care Behavior questionnaire was used to score compliance; lower scores indicated better self-care.

Results showed that 1 month after the intervention, the mean score of self-care behaviors was significantly improved in the intervention group when compared to the control group (Negarandeh et al., 2019).

Sayer et al. (2020) detailed the adoption of telehealth and restructured outpatient care with a shared clinic model at the New York Presbyterian Hospital in response to the SARS-COV-2 pandemic restrictions in patient care. Short term changes included the broad use of telehealth to provide ongoing patient care, the development of a centralized heart failure monitoring program to follow at risk patients remotely, and the creation of an outpatient practice model with a single physician providing in-person visits for whole group's patients. Long term changes resulted in a significant proportion of patients visits via telehealth visits and the expansion of a centralized heart failure program to provide comprehensive monitoring and treatment services (Sayer et al., 2020). Data on clinical 
outcomes were not reviewed in this article but were useful in aiding the DNP project plan and assessment of necessary resources.

Orso et al. (2020) developed and implemented a standardized questionnaire used during scheduled telephone visits to evaluate clinical stability. The Heart Failure Outpatient Clinic Telephonic Consulting Questionnaire evaluated important illness markers including social and functional condition, mood, medication- and vital monitoring adherence, and the current medical regimen. Answers to questions were given a score from 1 to 3 and follow up was scheduled based the sum of their scores. These authors concluded that the optimization of guideline-directed medical therapies improved symptoms in several patients who obtained a score reduction. Secondly, providers were able to identify poorly-adherent patients and provide regimen reinforcement (Orso et al., 2020).

Fersia et al. (2020) studied the impact on cardiology services and clinical activity during the SARS-COV-2 pandemic. Their assessment revealed fewer complaints of chest pain presentations and admissions, fewer cardiology referrals, and a substantial increase in use of virtual clinics during the first and second month of the lockdown. Although they did not assess for clinical improvement or whether their transition to virtual visits improved outcomes, it revealed that utilization of current technology and quick transition to virtual clinics were possible and positively received by both patients and providers (Fersia et al., 2020).

J. Lee et al. (2019) established a transitional care clinic that included early postdischarge follow up ( $<7$ days), phone call reminders prior to follow-up appointment, a clinical pharmacist for a medication reconciliation, and nurse practitioner involvement to 
address symptoms, monitor vital signs, schedule additional appointments, and provide medication compliance and lifestyle change education. Their study concluded that the transitional care clinic significantly reduced the rate of CHF-related readmissions within 7 days and 30 days of initial discharge; the most important aspect of reducing costly readmissions was ensuring timely follow up.

\section{Choosing the Heart Failure Outpatient Screening Tool}

The Heart Failure Outpatient Telephonic Consulting questionnaire was selected to create a standardized structure for follow-up visits. A downloadable PDF form of the questionnaire was obtained using a link taken from the "Protocol for Telehealth Evaluation and Follow-up of Patients with Chronic Heart Failure During the SARSCOV-2 Pandemic" (Figure 1; Orso et al., 2020). Data to be collected would include interviewee identifiers, social identifies, activities of daily living abilities, medications, vital signs (e.g., blood pressures, weights), current symptoms if any, and Sars-CoV-2 results. The data collected would have a corresponding number value; the total value classify the patient as green, yellow, or red, representing a suggested timeline for follow up. Utilizing this method during follow ups would allow standardization of important data collection to determine disease status and allow for identification of patients in need of management modifications. 


\section{Figure 1}

Heart Failure Outpatient Telephonic Consulting Questionnaire - Used with permission

\section{HF - OUTPATIENT CLINIC TELEPHONIC CONSULTING}

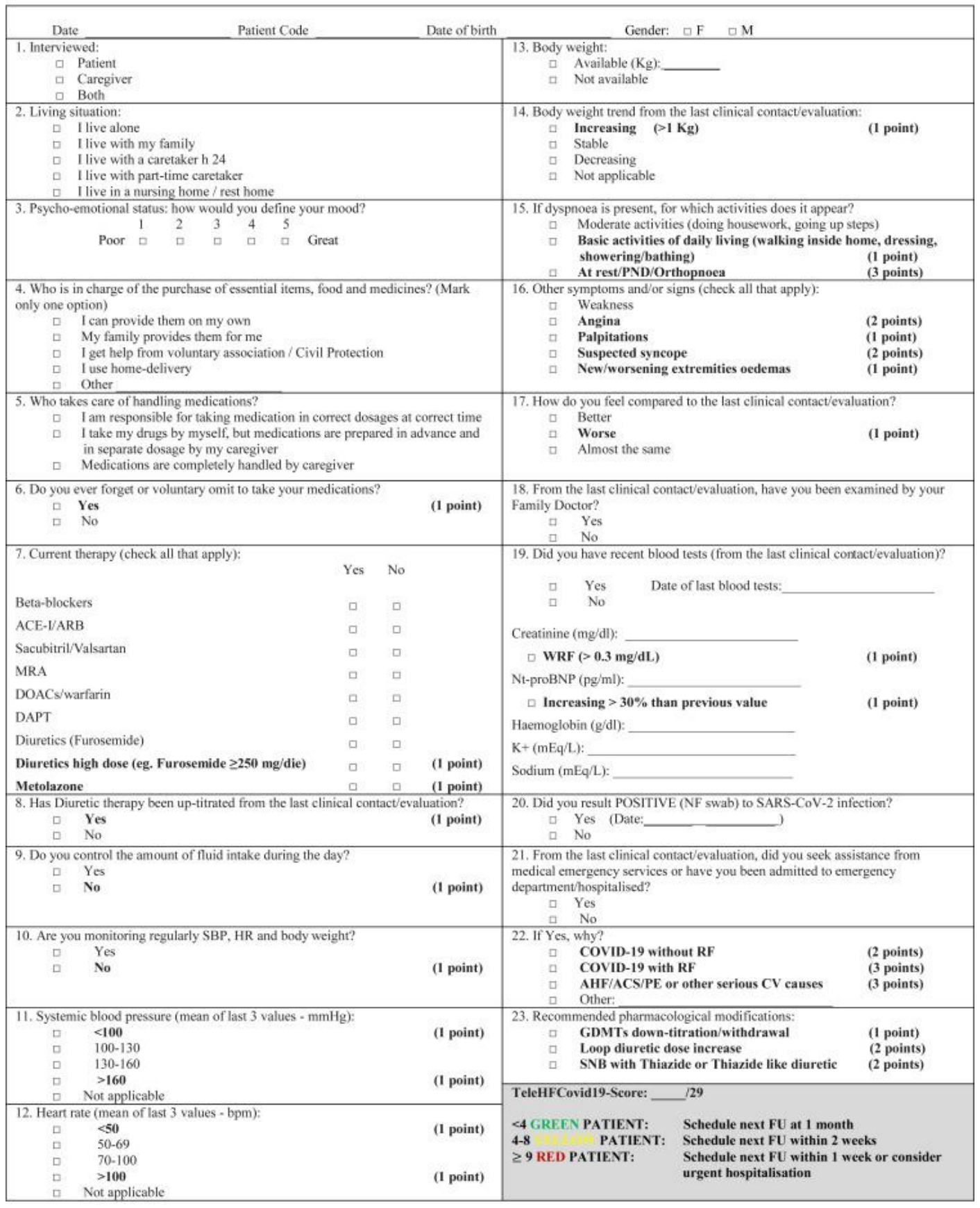

Next follow-up (date):

Hospitalisation recommended: Yes $\square$ No $\square$

Note. From "Protocol for Telehealth Evaluation and Follow-up of Patients with Chronic Heart Failure During the COVID-19 Pandemic, by F. Orso et al, 2020, Journal of the American Medical Directors Association, 21(12), https://doi.org/10.1016/j.jamda.2020.10.017. Reprinted with permission. 


\section{Program Development}

The proposed project includes a three-phase intervention: (a) chart review of the hospital course, disposition, and assessment of telemedicine follow-up barriers, (b) a telehealth assessment within 1 week of discharge using a Heart Failure Outpatient Clinic Telephonic Consulting Questionnaire (Orso et al., 2020) to assess for progress or deterioration, and (c) a final 4-week assessment using the heart failure questionnaire to review progress and assess readiness for cardiac rehab enrollment. Utilizing a standardized questionnaire would allow for assessment consistency throughout the entire project. Patients would be referred to the program by the hospital referral program using nursing staff and the heart-failure coordinator rounding. Inclusion to the project would require access to a method of telehealth services, either virtual video or telephone services. Not included in the project would be patients who were discharged home on hospice services and those who did not have access to telehealth modalities. Patients scores during the initial and follow up appointments would be compared to assess the effectiveness of their discharge management regimens.

\section{Project Intervention Process Next Steps}

IRB approval was received for this project (Appendix A). A future DNP student or practicing APRN could implement this intervention by collecting site-specific data, assessing clinic needs, and arranging transfer of this project and approval. Utilizing the heart failure outpatient screening tool, the future project will extract data regarding current patient status after discharge, Visit 2, and Visit 3. Results of the next stage would be discussed with a treating physician. Any health deteriorations, gaps in care, or modifiable medical therapies would be implemented and reassessed by the APRN at the 
next scheduled telehealth follow up appointment. Final data would be analyzed to demonstrate that an evidenced-based, APRN-lead, structured telephone assessment and follow up increases patient access to care and decreases 30-day hospital readmissions related to heart failure exacerbation.

Figure 2

Project Plan and Process

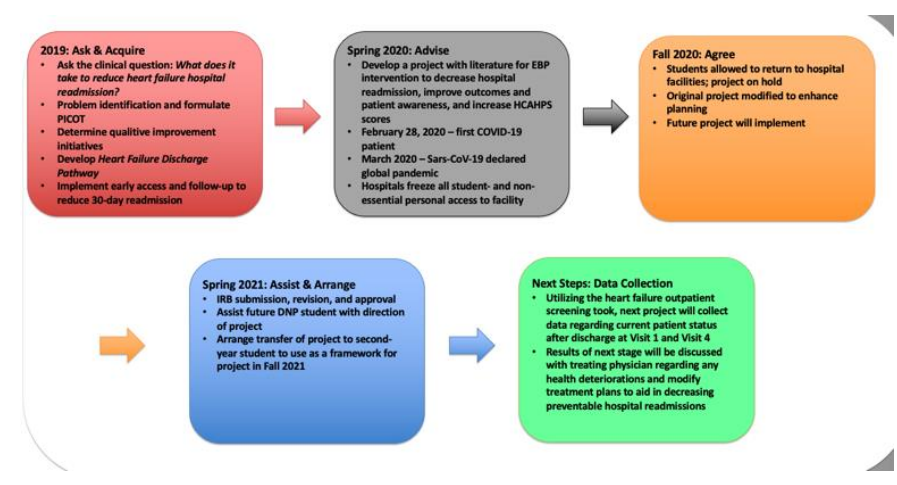

Project Analysis

This DNP project began in 2019 with the clinical question: What does it take to prevent a heart failure hospitalization? Months of literature review and multiple discussions with members of the heart failure team ensued during the project inception year. Due to the social-distancing restrictions and limitations caused by the SARS-COV2 pandemic, the project could not be completed. In Fall 2020, the original project was revised to include current best practices and a framework was created to make recommendations for the completion of this project by a future DNP student. Next steps of the project include submitting a separate IRB application for the new student, initiating follow up with heart failure patients with subsequent data collection, and formulating a final consensus as to whether the use of a structured telephone assessment with a standardized questionnaire decreased the incidence of heart failure readmissions. 


\section{Cost Benefit Analysis}

A cost-benefit analysis was limited by the inability to execute the entire project to completion. An estimation of cost benefit was made by researching the average cost of a heart failure hospitalization, maximum Medicare readmission penalties, and a factored cost for APRN time spent. The average medical cost for a heart failure hospitalization is $\$ 24,383$ per patient (Urbich et al., 2020). The hospital readmission reductions program lists a maximum penalty of no more than $3 \%$ reduced payment for a hospital readmission (Centers for Medicare \& Medicaid Services, 2020). Extensive online research gave a wide salary range for unexperienced nurse practitioners from \$56 to \$71 (Glassdoor, n.d.). A midrange figure of $\$ 67$ per hour was chosen for this analysis.

The three-step intervention would take 1 hour of the APRN's time totaling \$201. The costs of office space, internet, and telemedicine tools would not apply as they are already a part of care in most clinics and hospitals after the pandemic. A maximum total reduction of $3 \%$ penalty from a hospital readmission would decrease the maximum potential reimbursement by $\$ 732$ per readmission. For every hospitalization avoided, the net revenue would improve by $\$ 531$.

Total cost-benefit analysis shows that for every $\$ 1.00$ spent there is a $\$ 2.64$ savings or a $164 \%$ return of investment with the initiation of the APRN led telehealth intervention. The cost benefit analysis also considered financial, non-financial, and intangible benefits. Financial benefits include a reduction of readmission penalty charges, improved HCAPS scores for reimbursement purposes, income gained for the medical group by charging for APRN visits, and potential added income from community provider referrals to the hospital for patient care, cardiovascular exams, and cardiac 
rehabilitation enrollment. Non-financial benefits include improved patient satisfaction in care, patient retention and loyalty. Intangible benefits aim to improve cardiac patient outcomes, improve patient access to care after a discharge, and streamline services to the outpatient cardiology clinic and cardiac rehab.

\section{Recommendations}

Based on the literature review, the following recommendations were made:

1. Use structured telephone support to improve health related quality of life, increase direct access to health care providers, and provide more opportunities for disease and mediation education.

2. Use a standardized questionnaire during telemedicine appointments for the consistent identification of clinical stability and the patient's-adherence to the plan of care as well as to optimize guideline-directed medical therapies.

\section{Implications for Clinical Practice}

Optimizing modifiable symptoms is paramount to transitioning home from the hospital successfully. A standardized, structured telephone support can improve healthrelated quality of life, increase direct access to health care providers, provide more opportunities for disease- and medication education, and ultimately reduce preventable hospital readmissions. Increasing access through telehealth services will remove barriers in rural areas or those patients with transportation issues and can increase assessment frequency without the burden of multiple clinic visits. Although using the heart failure outpatient screening tool is neither prognostic nor validated, it can be useful in standardizing care by identifying deterioration, observing score reductions or increases, and suggesting appropriate reevaluation times. Utilizing best practices can optimize 
modifiable symptoms for a successful transition home from an in-patient hospital stay by increasing access to care, exposing patients to more frequent education and disease awareness strategies, and moving towards the goal of reducing hospital readmissions.

\section{Conclusion}

Congestive heart failure readmissions within 30 days of discharge have been a leading cause for quality initiative protocols. The Sars-CoV-19 pandemic has affected access to these patients in unforeseen ways. The swift transition to telehealth was a pivotal change in the management of heart failure patients after a discharge. These patients are known to deteriorate quickly necessitating strategies to ease accessibility and improve the quality of health care immediately after discharge. Multiple studies have demonstrated that early follow up, within the first 7 days of a hospital discharge, lowers the risk of hospital readmission within 30 days. Utilizing structured telephone support and a standardized telephonic questionnaire will provide consistency and identify necessary modifications to a patient's plan of care. APRNs will be instrumental in closing these relevant health care gaps. 


\section{References}

American Heart Association. (2018, June 13) Target: HF strategies and clinical tools. Retrieved April 3, 2021, from https://www.heart.org/en/professional/qualityimprovement/target-heart-failure/strategies-and-clinical-tools

Blum, M. R., Øien, H., Carmichael, H. L., Heidenreich, P., Owens, D. K., \& GoldhaberFiebert, J. D. (2020). Cost-effectiveness of transitional care services after hospitalization with heart failure. Annals of Internal Medicine, 172(4), 248-257. https://doi.org/10.7326/M19-1980

Centers for Medicare \& Medicaid Services. (2020, August 24). Hospital Readmissions Reduction Program (HRRP). Retrieved February 20, 2021, from https://www.cms.gov/Medicare/Medicare-Fee-for-ServicePayment/AcuteInpatientPPS/Readmissions-Reduction-Program

Dang, D., \& Dearholt, S. L. (Eds.). (2017). Johns Hopkins nursing evidence-based practice: Model and guidelines (3rd ed.). Sigma Theta Tau International.

Feltner, C., Jones, C. D., Cené, C. W., Zheng, Z.-J., Sueta, C. A., Coker-Schwimmer, E. J. L., Arvanitis, M., Lohr, K. N., Middleton, J. C., \& Jonas, D. E. (2014). Transitional care interventions to prevent readmissions for persons with heart failure: A systematic review and meta-analysis. Annals of Internal Medicine, 160(11), 774-784. https://doi.org/10.7326/M14-0083

Fersia, O., Bryant, S., Nicholson, R., McMeeken, K., Brown, C., Donaldson, B., Jardine, A., Grierson, V., Whalen, V., \& Mackay, A. (2020). The impact of the COVID-19 pandemic on cardiology services. Open Heart, 7(2), e001359.

https://doi.org/10.1136/openhrt-2020-001359 
Glassdoor. (n.d.). Sharp HealthCare Nurse Practitioner Hourly Pay. Retrieved March 11, 2020, from https://www.glassdoor.com/Hourly-Pay/Sharp-HealthCare-NursePractitioner-Hourly-Pay-E19168_D_KO17,35.htm

Lee, J., Reyes, F., Islam, M., Rahman, M., Ramirez, M., Francois, J., \& McFarlane, S. I. (2019). Outcomes of a transitional care clinic to reduce heart failure readmissions at an urban academic medical center. International Journal of Clinical Research \& Trials, 4, IJCRT-140. https://doi.org/10.15344/2456-8007/2019/140

Lee, K. K., Thomas, R. C., Tan, T. C., Leong, T. K., Steimle, A., \& Go, A. S. (2020). The Heart Failure Readmission Intervention by Variable Early Follow-up (THRIVE) study. Circulation: Cardiovascular Quality and Outcomes, 13(10).

\section{https://doi.org/10.1161/CIRCOUTCOMES.120.006553}

Negarandeh, R., Zolfaghari, M., Bashi, N., \& Kiarsi, M. (2019). Evaluating the effect of monitoring through telephone (tele-monitoring) on self-care behaviors and readmission of patients with heart failure after discharge. Applied Clinical Informatics, 10(2), 261-268. https://doi.org/10.1055/s-0039-1685167

Orso, F., Migliorini, M., Herbst, A., Ghiara, C., Virciglio, S., Camartini, V., Tognelli, S., Lucarelli, G., Fortini, G., Pratesi, A., Mossello, E., Di Bari, M., Marchionni, N., Ungar, A., Baldasseroni, S., \& Fattirolli, F. (2020). Protocol for telehealth evaluation and follow-up of patients with chronic heart failure during the COVID19 pandemic. Journal of the American Medical Directors Association, 21(12), 1803-1807. https://doi.org/10.1016/j.jamda.2020.10.017

Sayer, G., Horn, E. M., Farr, M. A., Axsom, K., Kleet, A., Gjerde, C., Latif, F., Sobol, I., Kelley, N., Lancet, E., Halik, C., Takeda, K., Naka, Y., Yuzefpolskaya, M., 
Kumaraiah, D., Colombo, P. C., Maurer, M. S., \& Uriel, N. (2020). Transition of a large tertiary heart failure program in response to the COVID-19 pandemic:

Changes that will endure. Circulation: Heart Failure, 13(9). https://doi.org/10.1161/CIRCHEARTFAILURE.120.007516

Strauss, S. E., Glasziou, P., Richardson, W. S., \& Haynes, R. B. (2019). Evidence-based medicine: How to practice and teach EBM (5th ed.). Elsevier.

Tersalvi, G., Winterton, D., Cioffi, G. M., Ghidini, S., Roberto, M., Biasco, L., Pedrazzini, G., Dauw, J., Ameri, P., \& Vicenzi, M. (2020). Telemedicine in heart failure during COVID-19: A step into the future. Frontiers in Cardiovascular Medicine, 7, 612818. https://doi.org/10.3389/fcvm.2020.612818

Urbich, M., Globe, G., Pantiri, K., Heisen, M., Bennison, C., Wirtz, H. S., \& Di Tanna, G. L. (2020). A systematic review of medical costs associated with heart failure in the USA (2014-2020). PharmacoEconomics, 38(11), 1219-1236. https://doi.org/10.1007/s40273-020-00952-0

Wosik, J., Clowse, M. E. B., Overton, R., Adagarla, B., Economou-Zavlanos, N., Cavalier, J., Henao, R., Piccini, J. P., Thomas, L., Pencina, M. J., \& Pagidipati, N. J. (2021). Impact of the COVID-19 pandemic on patterns of outpatient cardiovascular care. American Heart Journal, 231, 1-5. https://doi.org/10.1016/j.ahj.2020.10.074 
